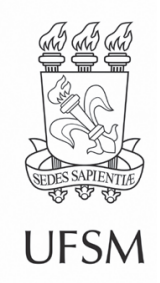

\title{
Artigos
}

\section{Sistemas silvipastoris com eucalipto: estocagem de carbono em diferentes espaçamentos e clones}

\author{
Silvopastoral systems with eucalypt: carbon storage in different spacing \\ and clones
}

\author{
Bruno Leão Said Schettini ${ }^{\circledR}{ }^{~}$, Laércio Antônio Gonçalves Jacovine ${ }^{\dagger}$, \\ Carlos Moreira Miquelino Eleto Torres ${ }^{\oplus}{ }^{\oplus}$, Silvio Nolasco de Oliveira Neto ${ }^{\oplus}$, \\ Samuel José Silva Soares da Rocha' ${ }^{\oplus}$, Paulo Henrique Villanova' ${ }^{\oplus}$, \\ Eliana Boaventura Bernardes Moura Alves ${ }^{1}$, \\ Maria Paula Miranda Xavier Rufino ${ }^{\oplus}$ \\ 'Universidade Federal de Viçosa, Viçosa, MG, Brasil
}

\section{RESUMO}

O objetivo do presente estudo foi comparar o uso de diferentes espaçamentos e clones de eucalipto na estocagem de carbono em 6 (seis) sistemas silvipastoris (SSP), localizados em Porto Firme - MG. Em campo foram utilizados experimentos contendo 6 (seis) SSP, com os clones GG100, 1144, 1224, híbridos de Eucalyptus grandis x Eucalyptus urophylla, nos espaçamentos de $6 \mathrm{~m} \times 4 \mathrm{~m}$ e $8 \mathrm{~m} \times 4 \mathrm{~m}$, nas idades de 60 e 96 meses. O inventário florestal realizado foi do tipo censo, onde todas as árvores tiveram sua circunferência a 1,30 m de altura (CAP) mensuradas e foram separadas em classes diamétricas. 0 volume foi estimado baseado no modelo Spurr. A densidade da madeira foi calculada pelo método de imersão em água e o estoque de carbono pelo fator de 0,47 . O material genético I224, no espaçamento de $6 \mathrm{~m} \times 4 \mathrm{~m}$, foi aquele com maior potencial de estocagem de carbono. A densidade básica para os três clones, no espaçamento de $6 \mathrm{~m} \times 4 \mathrm{~m}$, não variou estatisticamente. Porém, no espaçamento de $8 \mathrm{~m}$ x 4m, para os materiais genéticos GG100 e I224, houve diferença. Foi possível concluir que o material genético 1224, no espaçamento $6 \mathrm{~m} \times 4 \mathrm{~m}$ ( $24 \mathrm{~m}^{2}$ por planta) possui o maior potencial de estocagem de carbono e que os SSP com eucalipto contribuem para uma agricultura de baixa emissão de carbono e mitigação das mudanças climáticas.

Palavras-chave: Dados dendrométricos; Densidade da madeira; Espaçamento; Mudanças climáticas 


\section{ABSTRACT}

This study aimed to compare the use of different eucalypt spacing and clones in carbon storage in six silvopastoral systems (SSP) located in Porto Firme - MG. Experiments containing six SSP were used in the field, with clones GG100, 1144, and I224, hybrids of Eucalyptus grandis x Eucalyptus urophylla, in the spacing of $6 \mathrm{~m} \times 4 \mathrm{~m}$ and $8 \mathrm{~m} \times 4 \mathrm{~m}$, at the ages of 60 and 96 months. The forest inventory carried out was of the census type, where all the trees had their circumference at 1,30 $\mathrm{m}$ in height (CAP) measured and separated into diametric classes. The volume was estimated based on the Spurr model. The wood density was calculated by the method of immersion in water and the carbon stock by the factor of 0,47 . The genetic material 1224, spaced $6 \mathrm{~m} \times 4 \mathrm{~m}$, had the highest potential for carbon storage. The basic density for the three clones, in $6 \mathrm{~m} \times 4 \mathrm{~m}$ spacing, did not vary statistically, however, in $8 \mathrm{~m} \times 4 \mathrm{~m}$ spacing, for the genetic materials GG100 and I224, there was a difference. It was possible to conclude that the genetic material 1224 , in $6 \mathrm{~m} \times 4 \mathrm{~m}$ spacing ( $24 \mathrm{~m}^{2}$ per plant), has the highest potential for carbon storage and that SSP with eucalypt contributes to low-carbon agriculture and mitigation of climate change.

Keywords: Dendrometric database; Wood density; Spacing; Climate change

\section{INTRODUÇÃo}

O aumento das emissões dos principais gases de efeito estufa (GEE) na atmosfera $\left(\mathrm{CH}_{4}, \mathrm{CO}_{2}\right.$ e $\left.\mathrm{N}_{2} \mathrm{O}\right)$ provoca as mudanças climáticas em escala global (VOLKOVA et al., 2019). A concentração dos GEE na atmosfera começou a aumentar drasticamente a partir da década de 1950, com a industrialização mundial, o que motivou as reuniões internacionais sobre o clima (JOHN et al., 2020), que são realizadas há mais de duas décadas (WANG et al., 2020), com resultados modestos nas metas de redução das emissões de GEE (LUCON et al., 2013).

Os líderes das nações mais importantes do mundo, na década de 1990, tentaram mudar esse cenário com o Protocolo de Kyoto, quando as nações ricas firmaram o compromisso de reduzir as emissões de GEE. O problema desse protocolo era que apenas os países desenvolvidos tinham a obrigação de reduzir suas emissões. Como resultado, o primeiro período do Protocolo de Kyoto foi encerrado em 2012 (MIYAMOTO; TAKEUCHI, 2019). 
Em 2009, o governo brasileiro, na Conferência das Partes (COP), publicou seu acordo voluntário para reduzir as emissões de GEE, com novas metas. Em 2012, os líderes das nações mais importantes começaram a redigir um novo acordo climático oficial e em 2015 assinaram o Acordo de Paris, no qual países ricos e pobres têm o objetivo de reduzir as emissões. A meta do governo brasileiro é reduzir as emissões de GEE em 37,9 - 40,1\% em relação às emissões de 2005. Para atingir essa meta são necessárias mudanças no setor de energia, parar o desmatamento ilegal na Floresta Amazônica e aumentar a agricultura de baixo carbono. Porém, embora seja um dos países desenvolvidos, o Brasil precisa de recursos para se converter em uma sociedade de desenvolvimento sustentável (BRASIL, 2015).

O setor florestal é importante para o cumprimento da meta, que inclui a proposta de acabar com o desmatamento ilegal da Amazônia até 2030 e o fortalecimento do Plano de Agricultura de Baixa Emissão de Carbono ( $A B C$ ). Esse plano visa incentivar a instalação de cinco milhões de hectares de sistemas agroflorestais (SAFs) com potencial de mitigação entre 18-22 $\mathrm{GgCO}_{2 \mathrm{e}}$ (BRASIL, 2015). Os sistemas silvipastoris (SSPs), incluídos nas modalidades dos SAFs, utilizam árvores, animais e pastagens de maneira simultânea (NAIR, 2014) e funcionam como potenciais sumidouros de carbono. SSPs são SAFs que podem reduzir e compensar as emissões de GEE do setor agrícola brasileiro, principalmente por meio da integração da pecuária com as árvores plantadas (GOMES et al., 2020).

Por isso, são necessários estudos relacionados ao estoque de carbono em SSP's que avaliem como os diferentes materiais genéticos e espaçamentos influenciam a estocagem de carbono. Dessa forma, o objetivo do presente estudo foi comparar diferentes clones de eucalipto e espaçamentos na estocagem de carbono em diferentes sistemas silvipastoris. 


\section{MATERIAL E MÉTODOS}

\subsection{Descrição da área de estudo}

O estudo foi realizado em 6 sistemas silvipastoris, em diferentes idades, em uma propriedade rural localizada na cidade de Porto Firme $\left(20^{\circ} 41^{\prime} 12,42^{\prime \prime S}\right.$ e $43^{\circ}$ 0'34,46" O), Minas Gerais, Brasil (Figura 1). O clima na região é do tipo Cwa, segundo o sistema de Köppen, ou seja, subtropical com inverno seco e verão quente e chuvoso (ROLIM et al., 2007). A precipitação média mensal na região é de $110 \mathrm{~mm}$ e o total anual de $1.373 \mathrm{~mm}$.

Figura 1 - Localização dos sistemas silvipastoris em Porto Firme, Minas Gerais, Brasil

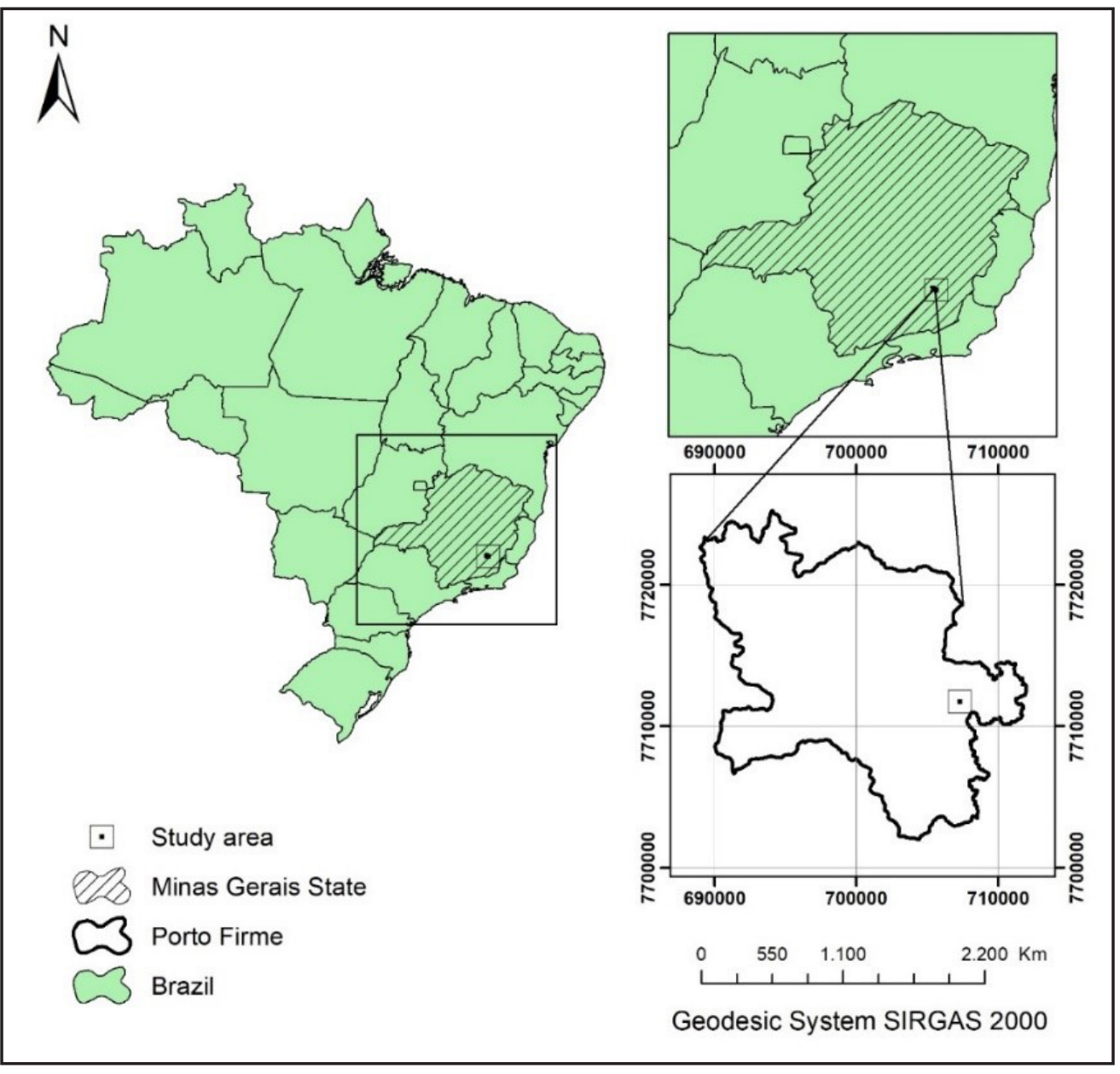

Fonte: Autores (2021) 
O componente arbóreo, em todos os sistemas, foi formado por clones de Eucalyptus grandis x Eucalyptus urophylla; o componente forrageiro por Brachiaria decumbens; e o componente animal foi o gado de corte, na quantidade de um animal ha-1 (Tabela 1). A adubação de plantio foi feita com a aplicação de $200 \mathrm{~g}$ de NPK na formulação 6-30-6; e a adubação de cobertura, 60 dias após o plantio, com a aplicação de 200g de NPK na formulação 20-00-20. A adubação da pastagem foi de 2 ton ha-1 de calcário.

Tabela 1 - Caracterização dos sistemas silvipastoris estudados

\begin{tabular}{lccccc}
\hline Sistema & Espaçamento $(\mathbf{m})$ & Área útil por planta $\left(\mathbf{m}^{2)}\right.$ & Clone & Área (ha) & Idade (meses) \\
\hline 1 & $6 \times 4$ & 24 & GG100 & 1,70 & 96 \\
2 & $6 \times 4$ & 24 & 1144 & 0,85 & 96 \\
3 & $6 \times 4$ & 24 & 1224 & 1,13 & 96 \\
4 & $8 \times 4$ & 32 & GG100 & 3,10 & 60 \\
5 & $6 \times 4$ & 24 & 1144 & 2,94 & 60 \\
6 & $8 \times 4$ & 32 & 1224 & 3,60 & 96 \\
\hline
\end{tabular}

Fonte: Autores (2021)

\subsection{Inventário florestal, densidade da madeira e estocagem de carbono}

O diâmetro à altura do peito (DAP), a 1,30m do solo, foi obtido por meio de inventário florestal do tipo censo. Três árvores por centro de classe (que variam de 12 a 18 árvores por sistema, em função da distribuição diamétrica), amostragem usualmente utilizada para plantios clonais de Eucalipto, foram selecionadas por sistema silvipastoril e submetidas à cubagem rigorosa. O volume total com a casca das árvores foi determinado pelo método não destrutivo, aplicando-se sucessivamente a expressão de Smalian. Os diâmetros em alturas de 0; 0,3; 0,7; e 1,3 m foram mensurados até o limite de 6,5 cm com auxílio do pentaprisma de Wheeler; a partir desse valor o volume foi calculado considerando o restante do fuste como um cone. 
O volume de madeira foi calculado baseado em dois diferentes modelos, Schumacher \& Hall (1933) e Spurr (1955), de acordo com as seguintes equações:

$\mathrm{V}=\beta_{0} * \mathrm{DAP}^{\beta 1} * \mathrm{Ht}^{\beta 2}+\varepsilon$

Onde: $V$ - Volume total, em m3; $\beta_{0}, \beta_{1}, \beta_{2}$ - parâmetros do modelo; DAP-diâmetro a 1,30 $\mathrm{m}$ de altura, em cm; $\mathrm{Ht}$ - altura total da árvore, em $\mathrm{m}$ e $\varepsilon$ - erro aleatório.

$\mathrm{V}=\beta_{0} *\left(\mathrm{DAP}^{2} * \mathrm{Ht}\right) * \beta_{1}+\varepsilon$

Onde: $V$ - Volume total, em m3; $\beta_{0}$ e $\beta_{1}$ - parâmetros do modelo; DAP - diâmetro a 1,30 $\mathrm{m}$ de altura, em cm; $\mathrm{Ht}$ - altura total da árvore, em $\mathrm{m}$ e $\varepsilon$ - erro aleatório.

As análises estatísticas foram realizadas com o software estatístico R(R Core Team 2013). As melhores equações foram baseadas nas análises dos seguintes itens: $\mathrm{R}_{\text {2adj }}$ (coeficiente de determinação empírico ajustado) e $\mathrm{S}_{\mathrm{y} \cdot \mathrm{x}}$ erro relativo.

A densidade básica da madeira foi determinada a partir de amostras coletadas no DAP, em 5 árvores por classe de diâmetro, com auxílio de um trado manual. O método utilizado para determinar a densidade básica da madeira foi o método de imersão em água (ABNT, 2003). A biomassa do tronco foi obtida multiplicando o volume (com sua casca) pela densidade básica da madeira. O estoque de carbono no fuste da árvore de cada indivíduo foi obtido multiplicando-se os valores de biomassa pelo fator 0,47. A densidade da madeira e o estoque de carbono, em cada um dos sistemas, foi comparada pelo Teste de Tukey a nível de significância de 95\%.

\section{RESULTADOS E DISCUSSÃO}

\subsection{Características dos sistemas}

A distribuição diamétrica nos sistemas estudados foi similar, com a maioria das árvores presentes nos centros de classes de 22,5 e 27,5 cm. O sistema 6, com material genético 1224 no espaçamento 8x4, teve comportamento diferente, com a maioria dos indivíduos no centro de classe de 17,5 cm, o que se deve à competição dos indivíduos por luz e nutrientes. 
O comportamento dos indivíduos em cada um dos sistemas segue a distribuição normal, que é o esperado para plantios homogêneos clonais. Resultado similar ao do presente estudo foi observado por Oliveira Neto et al. (2010). Ao avaliarem a distribuição diamétrica de um povoamento de eucalipto, aos 32 meses de idade, em diferentes arranjos, constataram que em espaçamentos mais amplos há maior número de indivíduos pertencentes às maiores classes de diâmetro.

Figura 2 - Número de indivíduos nos centros de classe de cada um dos sistemas estudados

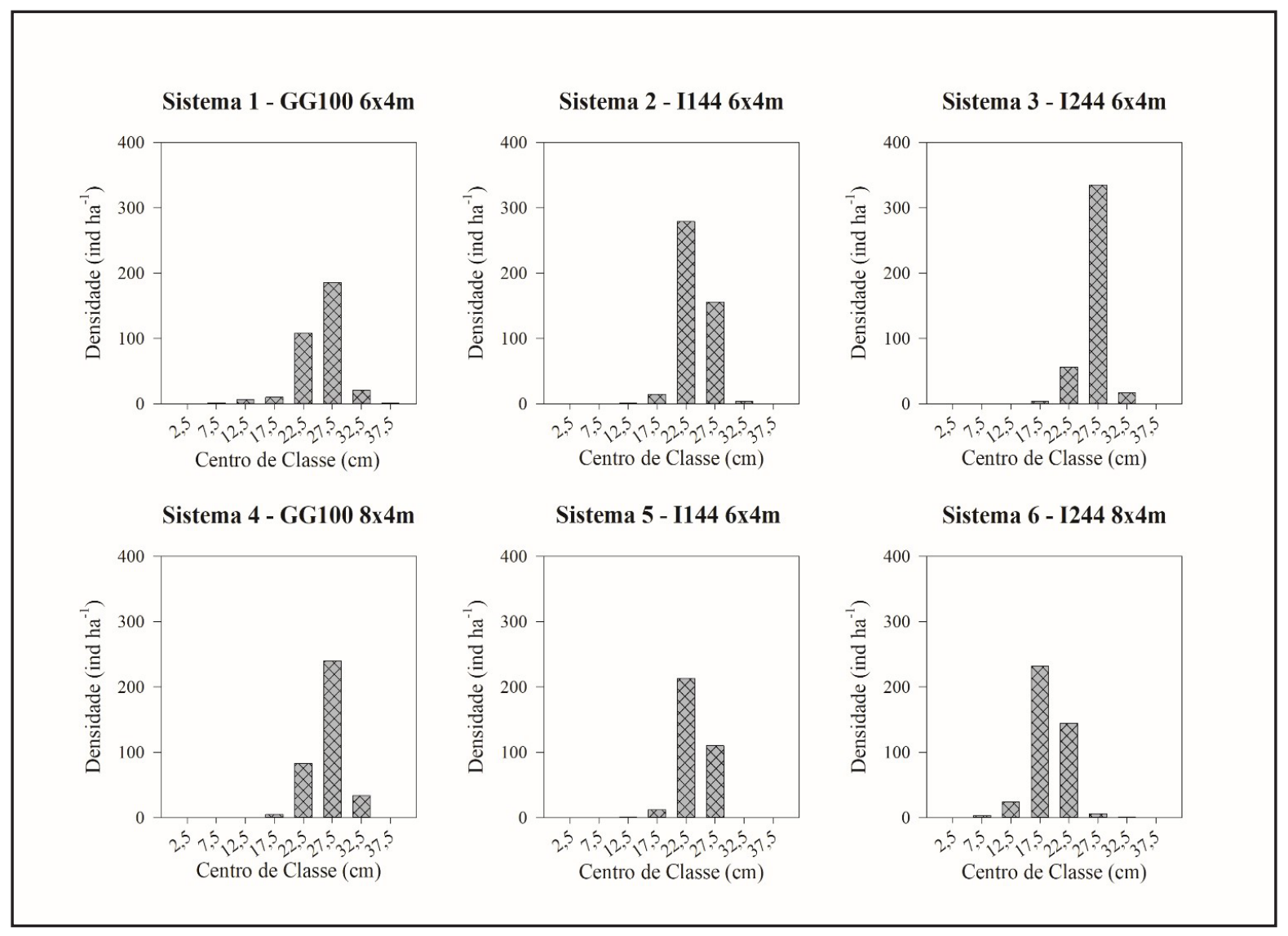

Fonte: Autores (2021)

\subsection{Escolha do modelo volumétrico a ser usado em cada sistema}

O modelo de Schumacher e Hall apresentou inconsistência, em 5 dos 6 sistemas avaliados, associadas ao parâmetro $\beta_{2^{\prime}}$ associado à variável altura total, o 
que impossibilita o seu uso. As equações utilizadas foram baseadas no modelo de Spurr, que apresentaram ajuste adequado, de acordo com a análise de $R_{2 \text { adj }}$ e $S_{y . x}$ e não tiveram inconsistências (Tabela 2).

Tabela 2 - Comparativo dos ajustes das equações volumétricas, baseadas nos modelos de Schumacher e Hall e Spurr, para os 6 sistemas avaliados

\begin{tabular}{|c|c|c|c|c|c|c|c|}
\hline Sistema & Clone & Modelo & $\beta_{0}$ & $\beta_{1}$ & $\beta_{2}$ & $\mathbf{R}_{\text {2adj }}(\%)$ & $S_{y \cdot x}$ \\
\hline \multirow[b]{2}{*}{1} & \multirow{2}{*}{ GG100 } & Schumacher & 0,000521 & 3,024481 & $-0,854753$ & 95,98 & 0,0845 \\
\hline & & Spurr & 0,000040 & 0,956600 & - & 89,43 & 0,1371 \\
\hline \multirow{2}{*}{2} & \multirow{2}{*}{1144} & Schumacher & 0,000255 & 2,705400 & $-0,345630$ & 95,58 & 0,0501 \\
\hline & & Spurr & 0,000143 & 0,829592 & - & 92,23 & 0,0665 \\
\hline \multirow{2}{*}{3} & \multirow{2}{*}{1224} & Schumacher & 0,000027 & 0,005648 & $-2,742000$ & 84,36 & 0,0765 \\
\hline & & Spurr & 0,000724 & 0,649855 & - & 84,06 & 0,0773 \\
\hline \multirow{2}{*}{4} & \multirow{2}{*}{ GG100 } & Schumacher & 0,000001 & 0,587600 & $-3,063000$ & 89,98 & 0,0734 \\
\hline & & Spurr & 0,000048 & 0,919400 & - & 89,44 & 0,0753 \\
\hline \multirow{2}{*}{5} & \multirow{2}{*}{1144} & Schumacher & 0,000240 & 2,650000 & $-0,325000$ & 99,17 & 0,1464 \\
\hline & & Spurr & 0,000050 & 0,910015 & - & 98,27 & 0,2273 \\
\hline \multirow{2}{*}{6} & \multirow{2}{*}{1224} & Schumacher & 0,000001 & 0,585600 & 3,063000 & 90,90 & 0,2005 \\
\hline & & Spurr & 0,000048 & 0,919400 & - & 90,40 & 0,2002 \\
\hline
\end{tabular}

Fonte: Autores (2021)

O valor negativo ao parâmetro $\beta_{2}$ impossibilita o uso do modelo de Schumacher e Hall, pois indica que quanto maior a altura da árvore, menor será o volume de madeira, o que caracteriza uma inconsistência biológica.

\subsection{Efeito do espaçamento na densidade da madeira e estocagem de carbono}

Não houve diferença estatística da densidade básica da madeira e estoque de carbono, quando comparada pelo Teste de Tukey, para o clone GG100 nos dois espaçamentos (Tabela 3). Cenário diferente do observado para o material genético 1224, em que houve diferença estatística. 
Diversos estudos foram realizados sobre o comportamento da densidade básica da madeira em plantios de eucalipto ao longo dos anos. Torres et al. (2016), ao compararem a densidade básica da madeira em sistemas silvipastoris em ViçosaMG, com diferentes arranjos espaciais, para um mesmo híbrido de Eucalyptus grandis x Eucalyptus urophylla, observaram que não houve diferença estatística entre os arranjos espaciais de $12 \times 3\left(0,43 \mathrm{~g} \mathrm{~cm}^{3-1}\right)$ e $8 \times 3 \mathrm{~m}\left(0,43 \mathrm{~g} \mathrm{~cm}^{3-1}\right)$, mas que eles diferiram estatisticamente pelo Teste de Tukey a 95\% de significância, quando comparados com o espaçamento de $9 \times 1 \mathrm{~m}\left(0,40 \mathrm{~g} \mathrm{~cm}^{3-1}\right)$. Essa diferença pode ser justificada pela maior competição por nutrientes em espaçamentos mais reduzidos.

Tabela 3 - Comparativo dos resultados obtidos nos sistemas 1, 4, 3 e 6 que possuem o mesmo material genético, porém em espaçamentos distintos

\begin{tabular}{lcccccccc}
\hline Sistema & Espaçamento & Clone & Volume & IMA & Densidade & Biomassa & Carbono & IMA $_{\mathrm{c}}$ \\
\hline 1 & $6 \times 4$ & GG100 & 186,51 & 23,31 & $0,4732 \mathrm{a}$ & 88,25 & 41,48 & $5,18 \mathrm{a}$ \\
4 & $8 \times 4$ & $\mathrm{GG100}$ & 193,70 & 24,20 & $0,4669 \mathrm{a}$ & 90,50 & 42,50 & $5,31 \mathrm{a}$ \\
3 & $6 \times 4$ & 1224 & 229,50 & 28,70 & $0,4641 \mathrm{a}$ & 106,50 & 50,10 & $6,26 \mathrm{a}$ \\
6 & $8 \times 4$ & 1224 & 125,18 & 25,04 & $0,3426 \mathrm{~b}$ & 42,80 & 20,12 & $3,35 \mathrm{~b}$ \\
\hline
\end{tabular}

Fonte: Autores (2021)

Em que: Espaçamento, em m; Volume, em $\mathrm{m}^{3} \mathrm{ha}^{-1} ; \mathrm{IMA}=$ Incremento médio anual, em $\mathrm{m}^{3} \mathrm{ha}^{-1}$ ano1; Densidade, em $\mathrm{g} \mathrm{cm}^{3-1}$; Biomassa, em ton ha-1; Carbono, em ton ha-1; e IMA ${ }_{c}=$ Incremento médio anual em carbono,em ton ha-1 ano-1. Médias seguidas de mesma letra, na coluna, não diferem entre si estatisticamente pelo Teste de Tukey a 5\% de significância.

O estoque de carbono não variou estatisticamente para o material genético GG100; resultado diferente ao observado para o clone 1224. Essa variação entre o estoque de carbono entre os dois materiais genéticos, nos diferentes espaçamentos, é explicada pela estagnação de crescimento do plantio ao longo dos anos. Villanova et al. (2018), ao avaliarem a curva de crescimento e estoque de carbono em 3 sistemas silvipastoris, na Zona da Mata de Minas Gerais, com diferentes híbridos de eucalipto (Eucalyptus urophylla $\mathrm{x}$ Eucalyptus grandis e Eucalyptus saligna), observaram que a idade 
de estabilização do crescimento em carbono variou entre 80 e 100 meses, para os diferentes sistemas, o que corrobora com o resultado do presente estudo.

Ao longo dos anos, a diferença entre os sistemas 1 e 4 pode ser maior, e o estoque de carbono no sistema 6 pode superar o do sistema 3, uma vez que arranjos espaciais mais amplos propiciam maior potencial de crescimento às plantas, em virtude da menor competição por recursos e nutrientes, o que ocasiona menor taxa de mortalidade e de árvores dominadas, sistemas silvipastoris com Eucalipto (SOUZA et al., 2020).

\subsection{Efeito do material genético de eucalipto na estocagem de carbono}

Não ocorreu diferença significativa na estocagem de carbono para os materiais genéticos avaliados (GG100 e I144) no espaçamento de 6 × 4 m. O estoque de carbono para os clones GG100 e 1224, no espaçamento de 8 x 4m, foram diferentes quando comparados pelo Teste de Tukey (Tabela 4).

Tabela 4 - Comparativo dos resultados obtidos nos sistemas 1, 2, 3, 4 e 6 que possuem o mesmo arranjo espacial, porém com materiais genéticos distintos

\begin{tabular}{lcccccccc}
\hline Sistema & Espaçamento & Clone & Volume & IMA & Densidade & Biomassa & Carbono & IMA $_{c}$ \\
\hline 1 & $6 \times 4$ & GG100 & 186,51 & 23,31 & $0,4732 \mathrm{a}$ & 88,25 & 41,48 & $5,18 \mathrm{a}$ \\
2 & $6 \times 4$ & 1144 & 205,20 & 25,70 & $0,4445 \mathrm{a}$ & 91,40 & 43,00 & $5,37 \mathrm{a}$ \\
3 & $6 \times 4$ & 1224 & 229,50 & 28,70 & $0,4641 \mathrm{a}$ & 106,50 & 50,10 & $6,26 \mathrm{~b}$ \\
4 & $8 \times 4$ & GG100 & 193,70 & 24,20 & $0,4669 \mathrm{a}$ & 90,50 & 42,50 & $5,31 \mathrm{a}$ \\
6 & $8 \times 4$ & 1224 & 125,18 & 25,04 & $0,3426 \mathrm{~b}$ & 42,80 & 20,12 & $3,35 \mathrm{~b}$ \\
\hline
\end{tabular}

Fonte: Autores (2021)

Em que: Espaçamento, em m; Volume, em $\mathrm{m}^{3} \mathrm{ha}^{-1} ; \mathrm{IMA}=$ Incremento médio anual, em $\mathrm{m}^{3} \mathrm{ha}^{-1}$ ano1; Densidade, em $\mathrm{g} \mathrm{cm}^{3-1}$; Biomassa, em ton ha- ${ }^{-1}$; Carbono, em ton ha-1; e IMA ${ }_{c}=$ Incremento médio anual em carbono, em ton ha-1 $\mathrm{ano}^{-1}$. Médias seguidas de mesma letra, na coluna, não diferem entre si estatisticamente pelo Teste de Tukey a 5\% de significância.

A maior concentração de indivíduos ha-1 no centro de classe de $27,5 \mathrm{~cm}$ no sistema 3 (material genético 1224) explica o maior volume de madeira deste sistema 
em relação aos sistemas 1 e 2, que tiveram comportamento similar na distribuição diamétrica. Apesar de não haver diferença estatística entre a densidade básica da madeira entre os três sistemas, a maior produção de madeira fez com que o estoque de carbono fosse superior no sistema 3, e diferente estatisticamente.

O estoque de carbono em um SSP com um híbrido Eucalyptus grandis $x$ Eucalyptus urophylla, no espaçamento de 8 × 3m, aos 44 meses, na região da Zona da Mata de Minas Gerais, teve o IMA ${ }_{c}$ de 3,13 ton ha-1 ano-1 $^{-1}$. Valor este inferior aos sistemas do presente estudo. Essa diferença pode ser justificada pelos diferentes espaçamentos e idades entre os SSP. O mesmo estudo avaliou o estoque de carbono em um sistema agrossilvipastoril (SASP), também com um híbrido de Eucalyptus grandis $x$ Eucalyptus urophylla, no espaçamento de $8 \times 3 \mathrm{~m}$, e o cultivo de feijão implantado no primeiro ano do sistema, com IMA $\mathrm{IM}_{\mathrm{c}}$ de 79 ton $\mathrm{ha}^{-1} \mathrm{ano}^{-1}$. A diferença de estoque de carbono entre o SSP e o SASP, segundo os autores, é decorrente dos restos culturais do feijão, presente na área após a colheita (CASTRO NETO et al., 2017). Em um SSP misto, aos 10 anos de idade, que o componente arbóreo foi dividido em Eucalyptus grandis e Acacia mangium, o estoque de carbono total foi de 14,29 ton $C$ ha $^{-1}$ (78,12\% presente no plantio de Eucalipto), o que evidencia a diferença de estocagem entre as espécies, que devem ser consideradas em função da destinação da madeira e tempo esperado para o retorno financeiro (MULLER et al., 2009).

Em sistemas silvipastoris, utilizando Eucalyptus grandis e Pinnus elliottii, no município de Alegrete, RS, aos 21 anos, o estoque de carbono, para densidade inicial de 500, 1.000 e 1.111 árvores por hectare de cada espécie, foi de 134,6; 150,8; e 182,9 ton $\mathrm{C}$ ha-1, respectivamente, resultando nos $\mathrm{IMA}_{\mathrm{c}}$ de 6,40, 7,18 e 8,70t $\mathrm{C}$ ha-1 $\mathrm{ano}^{-1}$ (OLIVEIRA, 2010). Esses valores são superiores aos encontrados no presente estudo, o que é justificado pela realização de desbastes (2 para o Pinus e 4 para o Eucalipto). Os desbastes favorecem o crescimento das árvores remanescentes no povoamento e aumento no estoque de carbono (MONTE et al., 2009).O estoque de carbono de 2 SSP, com híbridos de Eucalyptus grandis x Eucalyptus urophylla, no espaçamento de 9x1 e 
12x3m, foram capazes de neutralizar as emissões de GEE do sistema, o que ressalta a importância deste tipo de sistema no atual cenário brasileiro de uma agricultura com baixa emissão de GEE (TORRES et al., 2017).

Os SSP avaliados no presente estudo têm potencial de mitigação superior ao estabelecido pelo governo brasileiro na NDC do Acordo de Paris, o que ressalta a importância dos SSP no abatimento das metas brasileiras de redução das emissões de GEE. Os estoques de carbono dos sistemas estudados são similares entre si com o clone 1224 no espaçamento de $6 \times 4 \mathrm{~m}$ (24 $\mathrm{m}^{2}$ de área útil por planta) tendo o maior estoque de carbono - e com os estudos encontrados na literatura. Porém, o material genético e o arranjo espacial devem ser levados em consideração, pois têm influência sobre o estoque final de carbono do componente arbóreo. Os SAFs possuem contribuição para a estocagem de carbono, devendo, assim, serem incentivados pelo governo para auxiliar no cumprimento das metas de redução de emissões de gases de efeito estufa.

\section{CONCLUSÕES}

O material genético 1224, no espaçamento $6 \times 4 \mathrm{~m}\left(24 \mathrm{~m}^{2}\right.$ por planta), possui o maior potencial de estocagem de carbono para os clones de eucalipto avaliados.

Não existe diferença entre o potencial de estocagem de carbono dos materiais genéticos GG100 e I144, no espaçamento 6 × 4m (24m² por planta).

O material genético não influencia estatisticamente a densidade da madeira e estocagem de carbono para o clone GG100, nos espaçamentos de 6 x 4m (24m² por planta) e $8 \times 4 \mathrm{~m}\left(32 \mathrm{~m}^{2}\right.$ por planta).

O material genético influencia estatisticamente a densidade da madeira e estocagem de carbono para o clone 1224, nos espaçamentos de 6 x 4m (24m² por planta) e $8 \times 4 \mathrm{~m}\left(32 \mathrm{~m}^{2}\right.$ por planta).

O estoque de carbono dos 6 SSP's avaliados, com clones de eucalipto, é superior ao estabelecido pelo governo brasileiro no Acordo de Paris. 
Os estoques de carbono dos sistemas estudados são similares entre si e com os estudos encontrados na literatura. Porém, o material genético e o arranjo espacial devem ser levados em consideração, pois têm influência sobre o estoque final de carbono do componente arbóreo.

\section{REFERÊNCIAS}

ASSOCIAÇÃO BRASILEIRA DE NORMAS TÉCNICAS,ABNT.NBR. 16001. Responsabilidade socialsistema de gestão-requisitos. Rio de Janeiro: ABNT, 2003.

BRASIL. Pretendida contribuição nacionalmente determinada para consecução do objetivo da convenção-quadro das Nações Unidas sobre mudança do clima. Itamaraty, 2015. Disponível em: www.itamaraty.gov.br/images/ed_desenvsust/BRASIL-iNDC-portugues. pdf. Acesso em: 10 jan. 2016.

CASTRO NETO, F.; JACOVINE, L. A. G.; TORRES, C. M. M. E.; OlIVEIRA NETO, S. N.; CASTRO, M. M.; VILLANOVA, P. H.; FERREIRA, G. L. Balanço de Carbono - Viabilidade Econômica de Dois Sistemas Agroflorestais em Viçosa, MG. Floresta e Ambiente, v. 24, n. 1, p. 1-12, 2017. DOI 10.1590/2179-8087.092114

GOMES, F. J.; BOSI, C.; PEDREIRA, B. C.; SANTOS, P. M.; PEDREIRA, C. G. S. Parameterization of the APSIM model for simulating palisadegrass growth under continuous stocking in monoculture and in a silvopastoral system. Agricultural System, v. 184, n. 1, p. 102876, 2020. Disponível em: https://doi.org/10.1016/j.agsy.2020.102876. Acesso em: 11 dez. 2020.

JOHN, K.; JANZ, B.; KIESE, R.; WASSMANN, R.; ZAITSEV, A. S.; WOLTERS, V. Earthworms offset straw-induced increase of greenhouse gas emission in upland rice production. Science of the Total Environmental, v. 710, n. 1, p. 136352, 2020. Disponível em: https://doi.org/10.1016/j. scitotenv.2019.136352. Acesso em: 11 dez. 2020.

LUCON, O.; ROMEIRO, V.; PACCA, S. Reflections on the international climate change negotiations: A synthesis of a working group on carbon emission policy and regulation in Brazil. Energy Policy, v. 59, n. 1, p. 938-941, 2013. Disponível em: https://doi.org/10.1016/j.enpol.2013.04.058. Acesso em: 10 jan. 2016.

MIYAMOTO, M.; TAKEUCHI, K. Climate agreement and technology diffusion: Impact of the Kyoto Protocol on international patent applications for renewable energy Technologies. Energy Policy, v. 129, n. 1, p. 1331-1338, 2019. Disponível em: https://doi.org/10.1016/j. enpol.2019.02.053. Acesso em: 11 dez. 2020.

MONTE, M. A. et al. Crescimento de um clone de eucalipto submetido à desrama e desbaste. Revista Árvore, Viçosa, v. 33, n. 5, p. 777-787, 2009.

MÜLLER, M. D. et al. Estimativa de acúmulo de biomassa e carbono em sistema agrossilvipastoril na Zona da Mata Mineira. Pesquisa Florestal Brasileira, v. 60, n. 60, p. 11-17, 2009. 
NAIR, P. K. R. Agroforestry: Practices and Systems. Encyclopedia of Agriculture and Food Systems, v. 2. Amsterdam: Elsevier; 2014.

OLIVEIRA NETO, S. N. de et al. Crescimento e distribuição diamétrica de Eucalyptus camaldulensis em diferentes espaçamentos e níveis de adubação na região de Cerrado de Minas Gerais. Revista Floresta, v. 40, n. 4, p. 755-762, 2010.

OLIVEIRA, E. B. et al. Produção, Carbono e Rentabilidade Econômica de Pinus elliottii e Eucalyptus grandis em Sistemas Silvipastoris no Sul do Brasil. Pesquisa Florestal Brasileira, v. 57, n. 57, p. $45,2010$.

ROLIM, S. G.; DE CAMARGO, P. B. M.; LANIA, G. D.; DE MOARES, L. F. J. Classificação climática de Köppen e de Thornthwaite e sua aplicabilidade na determinação de zonas agroclimáticas para o estado de São Paulo. Bragantia, v. 66, n. 4, p. 711-720, 2007. Disponível em: https://doi. org/10.1590/S0006-87052007000400022. Acesso em: 10 jan. 2016.

SOUZA, H. S.; LOPES, A. S.; BARETTA, M. C.; SANTOS, J. O. P.; TSUKAMOTO FILHO, A. A. Crescimento de eucalipto em diferentes arranjos espaciais de sistemas silvipastoris no município de Santa Rita do Trivelato - Mato Grosso. Advanced in Forestry Science, v. 7, n. 1, p. 847-853, 2020. Disponível em: http://dx.doi.org/10.34062/afs.v7i1.5511. Acesso em: 11 dez. 2020.

TORRES, C. M. M. E.; JACOVINE, L. A.; OLIVEIRA NETO, S. N.; FRAISSE, C. W.; SOARES, C. P. B.; CASTRO NETO, F.; FERREIRA, L. R.; ZANUNCIO, J. C.; LEMES, P. G. Green house gas emissions and carbono sequestration by agroforestry systems in South Eastern Brazil. Scientific Reports, v. 7, n. 1, p. 16738, 2017. DOI 10.1038/s41598-017-16821-4

TORRES, C. M. M. E.; OLIVEIRA, A. C.; PEREIRA, B. L. C.; JACOVINE, L. A. G.; OLIVEIRA NETO, S. N.; CARNEIRO, A. C. O.; TORRES, C. M. M. E. Estimativas de produção e propriedades da madeira de eucalipto em Sistemas Agroflorestais. Scientia Forestalis, v. 44, n. 109, p. 137-148. DOI $10.18671 /$ scifor.v44n109.13

VILLANOVA, P. H.; JACOVINE, L. A. G.; TORRES, C. M. M. E.; ALVES, E. B. B. M.; OLIVEIRA NETO, S. N.; LEITE, H. G.; SCHETTINI, B. L. S.; ROCHA, S. J. S. S. Accumulation of carbon and age of thinning of the tree component in agroforestry systems. Revista Brasileira de Ciências Agrárias, v. 13, n. 2, p. 1-6, 2018. DOI10.5039/agraria.v13i2a5526

VOLKOVA, L.; ROXBURGH, S. H.; SURAWSKI, N. C.; MEYER, C. P.; WESTON, C. J. Improving reporting of national greenhouse gas emissions from forest fires for emission reduction benefits: An example from Australia. Environmental Science \& Policy, v. 94, n. 1, p. 49-62, 2019. Disponível em: https://doi.org/10.1016/j.envsci.2018.12.023. Acesso em: 11 dez. 2020.

WANG, X.; YU, Y.; LIN, L. Tweeting the United Nations Climate Change Conference in Paris (COP21): An analysis of a social network and factors determining the network influence. Online Social Networks and Media, v. 15, n. 1, p. 100059, 2020. Disponível em: https://doi. org/10.1016/j.osnem.2019.100059. Acesso em: 11 dez. 2020. 


\section{Contribuição de Autoria}

\section{1 - Bruno Leão Said Schettini}

Engenheiro Florestal, Me., Doutorando

https://orcid.org/0000-0001-6510-4511•bruno.schettini@ufv.br

Contribuição: Escrita - primeira redação, Escrita - revisão e edição, Conceituação, Curadoria de dados, Análise Formal, Investigação, Metodologia

\section{2 - Laércio Antônio Gonçalves Jacovine}

Engenheiro Florestal, Dr., Professor

https://orcid.org/0000-0001-5485-3090•jacovine@ufv.br

Contribuição: Conceituação, Análise Formal, Metodologia, Supervisão, Validação, Escrita - revisão e edição

\section{3 - Carlos Moreira Miquelino Eleto Torres}

Engenheiro Florestal, Dr., Professor

https://orcid.org/0000-0003-0255-2637•carlos.eleto@ufv.br

Contribuição: Conceituação, Análise Formal, Metodologia, Supervisão, Validação, Escrita - revisão e edição

\section{4 - Silvio Nolasco de Oliveira Neto}

Engenheiro Florestal, Dr., Professor

https://orcid.org/0000-0003-0182-5322•snolasco@ufv.br

Contribuição: Conceituação, Análise Formal, Metodologia, Supervisão, Validação, Escrita - revisão e edição

\section{5 - Samuel José Silva Soares da Rocha}

Engenheiro Florestal, Me., Doutorando

https://orcid.org/0000-0001-6686-1936•samuel.rocha@ufv.br

Contribuição: Investigação, Visualização de dados, Escrita - revisão e edição

\section{6 - Paulo Henrique Villanova}

Engenheiro Florestal, Me., Doutorando

https://orcid.org/0000-0002-4187-2740•paulo.villanova@ufv.br

Contribuição: Investigação, Visualização de dados, Escrita - revisão e edição 


\section{7 - Eliana Boaventura Bernardes Moura Alves}

Engenheira Florestal, Pesquisadora

https://orcid.org/0000-0003-2988-2345•eliana.moura@ufv.br

Contribuição: Investigação, Visualização de dados, Escrita - revisão e edição

\section{8 - Maria Paula Miranda Xavier Rufino}

Engenheira Florestal, Mestranda

https://orcid.org/0000-0001-6594-2152•maria.rufino@ufv.br

Contribuição: Investigação, Visualização de dados, Escrita - revisão e edição

\section{Como citar este artigo}

Schettini, B. R. L.; Jacovine, L. A. G.; Torres, C. M. M. E.; Oliveira Neto, S. N.; Rocha, S. J. S. S.; Villanova, P. H.; Alves, E. B. B. M.; Rufino, M. P. M. X. Sistemas silvipastoris com eucalipto: estocagem de carbono em diferentes espaçamentos e clones. Ciência Florestal, Santa Maria, v. 31, n. 3, p. 1047-1062, 2021. DOI 10.5902/1980509825281. Disponível em: https://doi. org/10.5902/1980509825281. Acesso em: xx mês-abreviado 2021. 